\title{
Analytical expression of pulse broadening in an arbitrary linear slow light medium
}

\author{
Miguel González-Herráez, ${ }^{1, *}$ Sonia Martin-Lopez, ${ }^{2}$ and Luc Thévenaz ${ }^{3}$ \\ ${ }^{1}$ Departamento de Electrónica, Universidad de Alcalá, Escuela Politécnica DO-231, 28871 Spain \\ ${ }^{2}$ Instituto de Óptica, CSIC. C/Serrano 144, Madrid 28006, Spain \\ ${ }^{3}$ EPFL Swiss Federal Institute of Technology, Institute of Electrical Engineering, Group for Fibre Optics, SCI-STI-LT Station11, \\ CH-1015 Lausanne, Switzerland \\ *Corresponding author: miguelg@depeca.uah.es
}

Received May 11, 2012; revised June 25, 2012; accepted June 25, 2012;

posted June 26, 2012 (Doc. ID 168398); published July 25, 2012

\begin{abstract}
We derive an analytical expression for the broadening of a Gaussian input pulse in an arbitrary linear slow light medium. The expression consists of two terms, one corresponding to amplitude broadening (low-pass filtering of the pulse bandwidth) and another corresponding to phase broadening (phase dispersion around the resonance). It is shown that for a Lorentzian gain profile, the amplitude broadening is dominant at small fractional delays. However, for large fractional delays, phase broadening is inevitably dominate. (c) 2012 Optical Society of America OCIS codes: $\quad 060.4370,290.5900,350.5500$.
\end{abstract}

In the last few years, the scientific community has shown a large interest in slow light systems. This interest has been mostly because of the possible use of slow light devices as optical buffers or optical delay lines [1] . A number of slow light systems have been experimentally realized based on different physical phenomena such as electromagnetically induced transparency, coherent population oscillations, optical parametric amplification, and stimulated scattering processes [2]. All these slow light schemes share a common feature making the essence of the slow and fast light generation in optical media: the presence of one or multiple strong spectral resonances to obtain a highly dispersive material. Unfortunately, this highly dispersive behavior is also accompanied with an inevitable distortion (in amplitude and phase) that mostly manifests as pulse broadening. As a result, the larger the delay in the slow light medium, the more broadening in the output pulse. In order to overcome this delay-distortion trade-off, several slow light schemes have been theoretically and experimentally investigated [2-9]. In particular, it was found that tailoring the shape of the spectral resonance to optimize the dispersive properties of the material could considerably reduce the induced distortion while keeping the fractional delay. However, even with this approach, the maximum achievable pulse delay was found to be limited to a few pulse durations. From the point of view of the applications, it is important to have simple analytical ways of evaluating the distortion introduced by an arbitrarily spectrally shaped slow light medium. This paper aims to provide a general method of evaluating the distortion in these arbitrary-shaped systems. The results of the paper may also be useful for filter design in telecommunication systems, where pulse distortion might be a key concern.

We provide here an analytical study of Gaussian pulse broadening propagating in a slow light medium presenting an arbitrary spectral transmission. The main result of the paper is an analytical expression that quantifies the total broadening accumulated by the Gaussian input pulse as a function of the higher order moments in the amplitude and phase response of the system under study.
We show that this broadening can be expressed as the vector sum of two components: one associated to distortion in the amplitude response of the system (amplitude broadening or low-pass filtering effect) and the other associated to the higher order dispersion terms in the phase response of the system (phase broadening). Our expression gives explicit forms for the amplitude and phase broadenings. We show that phase broadening dominates over amplitude broadening for large fractional delays. This is consistent with the ringing effect observed in many experiments attempting large fractional delays and also in clear opposition to the conventional view that strictly attributes the main source of broadening in slow light media to the low-pass filtering effect (amplitude distortion) $[\underline{1}, \underline{4}]$.

We began our derivation by finding a suitable description of any linear slow light system. Mathematically, a linear system can be completely characterized by its impulse response; i.e., by the output of the system when it is fed with a Dirac delta at the input. The response of the medium to an arbitrary input can then be calculated as the convolution of the input waveform with the impulse response. In the Fourier domain, the transform of the impulse response is given by the transfer function $H(\omega)$, and the output of the system $(\tilde{A}(\omega))$ for an arbitrary input $\left(S_{\text {in }}(\omega)\right)$ can be obtained as $\tilde{A}(\omega)=$ $H(\omega) \cdot S_{\text {in }}(\omega)$. In the particular case of material slow light systems (such as those based on stimulated Brillouin scattering [3-6]), the transfer function has the form $H(\omega)=\exp (g(\omega)+i \Phi(\omega))$, where $g(\omega)$ and $\Phi(\omega)$ are related by the well-known Kramers-Kronig (KK) relations. KK relations basically result from causality in the response of the system; i.e., the response of the system at any time $t=t_{1}$ depends only on the system input at previous times $t<t_{1}$.

In an ideal scenario, slow light systems should exhibit a flat amplitude response and a linear phase response with tunable slope, hence $g=g_{0}$ and $\Phi(\omega)=\Phi_{1} \omega$. While this response would be desirable, it also turns out to be impossible except using passive (nontunable) delaying elements with long propagation lengths. Tunable delaying elements [3- $\underline{3}$ ] may introduce significant delays 
at the expense of working close to or within a resonance. This means that the system operates only in a narrow frequency band (i.e., the system is bandwidth limited) and that the distortion in the signal is always present and should be restricted depending on the actual application requirements. This also implies that broadening is inevitable in linear slow light systems: any system that introduces a low-pass filtering on the signal (a reduction in the rms spectral width) always causes pulse broadening due to the time-frequency uncertainty principle [10]. This problem is conventionally known as the delay-bandwidth product limitation [11].

Linear slow light systems are tailored to have a symmetric spectral response around the central frequency of the pulse. In a resonance, the center is the region that exhibits larger delay with less distortion. As a result of the real impulse response, $g$ exhibits even symmetry, and consequently $\Phi$ exhibits odd symmetry. A series expansion of $g$ and $\Phi$ can be found:

$$
\begin{gathered}
g=g_{0}+g_{2} \omega^{2}+g_{4} \omega^{4}+\ldots \\
\Phi=\Phi_{1} \omega+\Phi_{3} \omega^{3}+\Phi_{5} \omega^{5}+\ldots
\end{gathered}
$$

where, for simplicity, the resonance is centered at $\omega=0$. In terms of a physical interpretation of the coefficients of the series expansion, $g_{0}$ can be interpreted as the amplitude gain/loss of the system in the center of the resonance and $\Phi_{1}$ is the delay associated to the slow light effect. All the higher order terms in the expansion are undesired (although mostly inevitable) and bring distortion in the pulse: The higher order terms in $g\left(g_{2}, g_{4}\right.$, etc) introduce amplitude distortion and the higher order terms in $\Phi\left(\Phi_{3}\right.$, $\Phi_{5}$, etc) introduce phase distortion. Equations (1) and (2) can be related by reverting to KK relations.

With regard to these expressions, it is important to have in mind two important concepts: (1) since $g$ and $\Phi$ contribute in the transfer function as purely real and imaginary components, respectively, their distortion effect can never mutually cancel (they add distortion geometrically, as a sort of Pythagorean sum), and (2) since $\Phi$ shows only odd terms in its expansion, its broadening effect cannot be compensated with conventional second-order chromatic dispersion (which even introduces more phase distortion) that would be represented by a $\Phi_{2}$ term.

Our procedure starts by defining a shape-independent metric of pulse width and pulse broadening. The best metric that we find is the rms pulse width:

$$
\sigma_{t}^{2}=\frac{1}{E} \int_{-\infty}^{+\infty} t^{2}|A(t)|^{2} \mathrm{~d} t
$$

where $A(t)$ is the temporal amplitude of the pulse at the output of the slow light system and

$$
E=\int_{-\infty}^{+\infty}|A(t)|^{2} \mathrm{~d} t
$$

is the total energy inside the pulse. In the above expressions, it has been assumed that both $A(t)$ and $t A(t)$ are absolutely integrable. For simplicity, we have considered that the pulse is always centered at $t=0$. This simplifies the relations, and the results do not lose any generality. The rms pulse width is the definition of pulse width that is conventionally used to quantify pulse distortion in fiber-optic communication systems. This definition of pulse width measures how much the pulse energy is temporally "spread" regardless of the shape.

We can connect these expressions of pulse broadening in the time domain to the frequency domain (where our slow light systems are better described) by using Parseval's theorem and the well-known properties of the Fourier transform [12]:

$$
\sigma_{t}^{2}=\frac{1}{E_{\omega}} \int_{-\infty}^{+\infty}\left|\frac{d \tilde{A}}{d \omega}\right|^{2} \mathrm{~d} \omega,
$$

where $\tilde{A}(\omega)$ is the Fourier transform of $A(t)$. Let us now consider that the Fourier transform of the pulse envelope reads $\tilde{A}(\omega)=X(\omega) \cdot \exp [i \varphi(\omega)]$, where $X$ and $\varphi$ are now purely real functions. In terms of the slow light system, we can recall that $\tilde{A}(\omega)=H(\omega) \cdot S_{\text {in }}(\omega)$; hence, $X(\omega)$ accumulates all the amplitude distortion introduced by the slow light system and $\varphi(\omega)$ accumulates all the phase distortion. We can finally obtain an expression that relates the pulse width to the amplitude and phase responses of the system $[\underline{10}, \underline{11}]$ :

$$
\begin{aligned}
\sigma_{t}^{2} & =\frac{1}{E_{\omega}}\left[\int_{-\infty}^{+\infty}\left|\frac{d X}{d \omega}\right|^{2} \mathrm{~d} \omega+\int_{-\infty}^{+\infty}|X(\omega)|^{2}\left|\frac{d \varphi}{d \omega}\right|^{2} \mathrm{~d} \omega\right] \\
& =\sigma_{X}^{2}+\sigma_{\varphi}^{2},
\end{aligned}
$$

where, for simplicity, it has been assumed that the mean group delay has been subtracted from the output pulse (as stated before, the pulse is centered in $t=0$ ). Equation (6) shows that there are two main contributions to the temporal broadening of the pulse: One is due to the variations in the spectral amplitude distribution in the signal $\left(\sigma_{X}\right)$, which can be modified through the slow light system by the spectral filtering effect, the so-called amplitude broadening, and the other $\left(\sigma_{\varphi}\right)$ is due to the variations of the spectral phase distribution in the pulse (weighted by the pulse energy) and can thus be related to the phase distortion introduced by the medium. As it is visible, both contributions are positive (they add geometrically), and therefore, they can enter into no compensation scheme. We can also see that the "amplitude" contribution to the pulse width is the only term that never vanishes unless the energy is zero or the pulse is spectrally flat (the pulse is a Dirac delta).

Now we can quantify the relative importance of each of these contributions in the broadening of a pulse traveling through a slow light medium. We consider then a Gaussian pulse at the input of the slow light system $s_{\text {in }}(t)=$ $\exp \left(-t^{2} / 2 \sigma_{\text {in }}^{2}\right)$ (defined in amplitude), whose Fourier transform is straightforwardly obtained: $S_{\text {in }}(\omega)=$ $\exp \left(-\omega^{2} \sigma_{\text {in }}^{2} / 2\right)$. For convenience in the mathematical formulation, we can rewrite the expression of $H(\omega)$ as $H(\omega)=G(\omega) \exp (i \Phi(\omega))$, where $G(\omega)$ can also be rewritten as a Taylor series expansion: 


$$
G=\exp \left(g_{0}\right)\left[1+g_{2} \omega^{2}+\left(g_{4}+\frac{g_{2}^{2}}{2}\right) \omega^{4}+\ldots\right],
$$

while expansion in $\Phi$ can be found in Eq. (2). We can now insert $H(\omega) S_{\text {in }}(\omega)$ in Eq. (6) and recall the properties of Gaussian integrals. An analytical expression can then be found for the amplitude and phase broadenings:

$$
\begin{gathered}
\sigma_{X}^{2}=\sigma_{\mathrm{in}}^{2} \frac{1-g_{2} \frac{1}{\sigma_{\mathrm{in}}^{2}}-\frac{\left(9 g_{4}+g_{2}^{2}\right)}{2} \frac{1}{\sigma_{\mathrm{in}}^{4}}+\ldots}{1+g_{2} \frac{1}{\sigma_{\mathrm{in}}^{2}}+\frac{3\left(g_{4}+g_{2}^{2}\right)}{2} \frac{1}{\sigma_{\mathrm{in}}^{4}}+\ldots} \\
\sigma_{\varphi}^{2}=\frac{27 \phi_{3}^{2}}{2 \sigma_{\mathrm{in}}^{4}} \frac{1+5 g_{2} \frac{1}{\sigma_{\mathrm{in}}^{2}}+\frac{35\left(g_{4}+g_{2}^{2}\right)}{2} \frac{1}{\sigma_{\mathrm{in}}^{4}} \ldots}{1+g_{2} \frac{1}{\sigma_{\mathrm{in}}^{2}}+\frac{3\left(g_{4}+g_{2}^{2}\right)}{2} \frac{1}{\sigma_{\mathrm{in}}^{4}}+\ldots}+\ldots,
\end{gathered}
$$

where, for brevity, we have just included terms up to the fourth order in amplitude distortion and third order dispersion in the phase distortion. Note that $\Phi_{1}$ has no impact on the pulse broadening, as it merely introduces a delaying effect.

We can now easily analyze these expressions. It can be clearly seen that the only way to have no distortion $\left(\sigma_{X} / \sigma_{\text {in }}=1, \sigma_{\varphi}=0\right)$ is to set all the coefficients other than $g_{0}$ to 0 . Otherwise, distortion is introduced in the pulse leading in most cases to broadening of the pulse. Retaining only the leading terms in the expansion, amplitude broadening is introduced whenever $g_{2}<0$; that is, the amplitude response of the system shows a low-pass signature. On the contrary, amplitude broadening can be made smaller than one for $g_{2}>0$ but this would normally favor phase broadening. This is a general trend in Eqs. (8) and (9); the interested reader may find out that similar conclusions could be obtained with $g_{4}$. What is convenient for minimizing amplitude broadening turns out to be detrimental for minimizing the phase broadening. Another interesting conclusion comes from analyzing the growth rates when attempting large fractional delays $\left(\Phi_{1} / \sigma_{\text {in }}\right)$. This can be simply evaluated in Eqs. (8) and (9) by keeping $\Phi_{1}$ and all other coefficients $g_{n}$ and $\Phi_{n}$ constant and decreasing $\sigma_{\text {in }}$. It can be seen that the growth rate of the phase broadening is much larger than the growth rate of the amplitude broadening. This is consistent with the ringing effects typically associated with third order dispersion, which has been observed in experiments attempting large fractional delays (see, for instance, [13]). To overcome this third order dispersion effect, a pure linear phase spectral response was recently proposed [14], which could be interesting for large fractional delays and moderate values of distortion.

We can now visualize this last conclusion by computing amplitude and phase broadenings in a typical slow light medium (a Lorentzian gain profile). We perform this computation using Eq. (6) for different widths of the input Gaussian pulse in relation to the inverse bandwidth of the slow light device $1 / \Delta$. The results are plotted in Fig. 1 for $g_{0}=5$ (21.7 dB gain in the signal). As could be expected, the broadening grows rapidly for pulses with a frequency bandwidth larger than the bandwidth of the slow light device. Generally, the amplitude contribution is

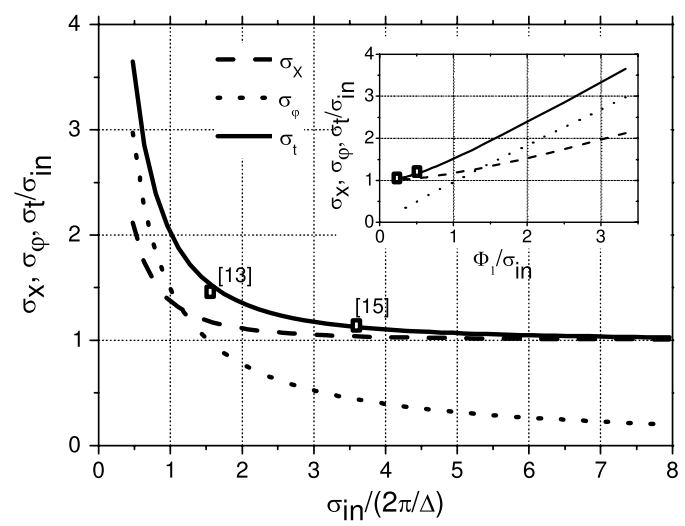

Fig. 1. Amplitude $\left(\sigma_{X}\right)$ and phase $\left(\sigma_{\varphi}\right)$ broadenings accumulated in a Gaussian pulse as a function of the input pulse width $\sigma_{\text {in }}$ normalized by the inverse bandwidth of the slow light system $2 \pi / \Delta$. Computation has been done using the exact expression for the Lorentzian gain and Eq. (6). Experimentally obtained points have been added for comparison (taken from [13] and [15]). Inset shows the same broadening data plotted versus fractional delay.

larger except when $\sigma_{\text {in }} /(2 \pi / \Delta)$ becomes less than 1.1 (in these conditions, this is equivalent to having a fractional delay greater than 1.6). It is noticeable that the growth rate of the phase broadening in this regime is larger than the growth rate of amplitude broadening in this same working region. This situation becomes more severe when attempting larger fractional delays.

In conclusion, we have provided an analytical expression that quantifies the total broadening accumulated by the Gaussian input pulse as a function of the higher order moments in the amplitude and phase response of the system. Our expression gives explicit forms for the amplitude and phase broadenings. We have also shown that phase broadening dominates over amplitude broadening for large fractional delays.

\section{References}

1. R. W. Boyd and D. J. Gauthier, in Progress in Optics, E. Wolf, ed. (Elsevier, 2002), Vol. 43, p. 497.

2. J. B. Khurgin, Adv. Opt. Photon. 2, 287 (2010).

3. M. D. Stenner, M. A. Neifeld, Z. Zhu, A. M. C. Dawes, and D. J. Gauthier, Opt. Express 13, 9995 (2005).

4. M. G. Herráez, K. Y. Song, and L. Thévenaz, Opt. Express 14, 1395 (2006).

5. A. Zadok, A. Eyal, and M. Tur, Opt. Express 14, 8498 (2006).

6. R. Pant, M. D. Stenner, M. A. Neifeld, and D. J. Gauthier, Opt. Express 16, 2764 (2008).

7. J. T. Mok, C. M. Sterke, I. C. M. Littler, and B. J. Eggleton, Nat. Phys. 2, 775 (2006).

8. S. Sandhu, M. L. Povinelli, M. F. Yanik, and S. Fan, Opt. Lett. 31, 1985 (2006).

9. J. B. Khurgin, Opt. Lett. 31, 948 (2006).

10. M. Gonzalez-Herraez and L. Thévenaz, Opt. Express 17, 4732 (2009).

11. D. A. B. Miller, Phys. Rev. Lett. 99, 203903 (2007).

12. L. Cohen, Time-Frequency Analysis (Prentice Hall, 1995).

13. K. Y. Song, M. G. Herraez, and L. Thevenaz, Opt. Lett. 30, 1782 (2005).

14. M. González-Herráez and L. Thévenaz, Proc. SPIE 7949, $79491 B$ (2011).

15. K. Y. Song, M. G. Herraez, and L. Thevenaz, Opt. Express 13, 82 (2005). 